\title{
Erratum
}

\section{Culturally Safe Falls Prevention Program for Inuvialuit Elders in Inuvik, Northwest Territories, Canada: Considerations for Development and Implementation-ERRATUM}

\author{
Julia S. Frigault and Audrey R. Giles
}

https://doi.org/10.1017/S0714980819000308. Published by Cambridge University Press, 25 June 2019

The Inuvialuit translation of the abstract was omitted from the original online version of the article by Frigault and Giles (published online 25 June 2019). It has been added to the online version and will be included in the printed version as well. The translation is as follows:

\section{INUVIALUIT TRANSLATION}

Using poscolonial tamna atughulugu kanuk pangma katagumagata ilthuakniahulugu

ukautigikangat tamani inunaktuat kataguruat katalaikulgi havatuat [LFPP] kataaipyakuvlugi utukanat tavraniituat lnuvialuit inuniaktuat luvingmi. tamanituat NWTmi Canatami iltchugilugu kanuk katangnik iltchugitkuvlgu havaktuat tamatkununga utukannait inuvialuit ikayukiguting tamakunuga havaktuanun tamani inuniaktuat pakitchuatguk utukananin tapkualu LFPP inuvingmi tamani nunami inuuniaktuat inuilu hutgautngnik kanuglu ilihautilugit utukanat tamaniittualu ilihautinik tavranituanik,tamanilu inuuniahynik nakuruanik havaunanik pilthanaitchuanik havaguting tamakunani LFPP sililugu ukpignaktuanikinilani tamnicgimaitchualu inuit katirutigilugu tana katangnatuak pitchilnak tamani ilihautigilugu nakuruanik hunaichuaanik ililugu ilipialugu tamanianugnaicguaniktamakunga niapiktuguruanun tamani utukanat

We regret the omission.

\section{Reference}

Frigault, J. S., \& Giles, A. R. (Published 25 June 2019). Culturally safe falls prevention program for Inuvialuit elders in Inuvik, Northwest Territories, Canada: Considerations for development and implementation. Canadian Journal on Aging, https:/ / doi. org/10.1017/S0714980819000308. 